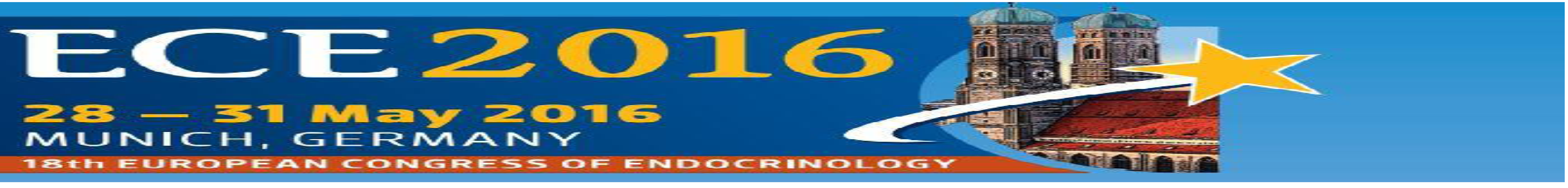

\title{
PSYCHIATRIC TROUBLES REVEALING ISOLATED PRIMARY HYPOALDOSTRONISM
}

\section{Boudiaf D* (Dr), Azzoug S (Pr), Chentli F (Pr)}

Department of Endocrinology and Metabolic diseases, Algiers, Algeria.

*Corresponding author: boudiaf.dia@outlook.com

Introduction : Hyponatremia's etiological diagnosis remains a challenge in the real life. Our aim was to report a patient who was sent for severe behavioral disorders revealing hyponatremia due to an isolated deficit in Aldosterone. This observation emphasizes the extreme rarity of the disease which deserves to be reported.

Case report: A young patient aged 34 with a history of autoimmune thyroiditis consulted for an acute and severe psychiatric syndrome with normal cerebral MRI. His clinical examination was normal except for some signs of dehydratation. Biological assessment revealed a profound hyponatremia $(<120 \mathrm{mmol} / \mathrm{l}, \mathrm{N}=135-145)$ which did not respond to conventional treatment.

Blood glucose and kidney function were normal. Glucocorticoids deficiency was excluded as several plasma cortisol were normal $(>500 \mathrm{nmol} / \mathrm{L}$ ). Faced to this enigma, isolated aldosterone deficiency was suspected and confirmed. Actually, aldosterone in standing position was low (39-56 pmol/L, N=208-1000) and plasma Renin was increased: $2139 \mathrm{mIU} / \mathrm{L}$ (N: 4.4 - 46.1). He was treated with Fludrocortisone and the follow up showed sodium normalization, spectacular improvement of the general condition, and disappearance of psychiatric troubles.

\section{Resistant Hyponatremia}

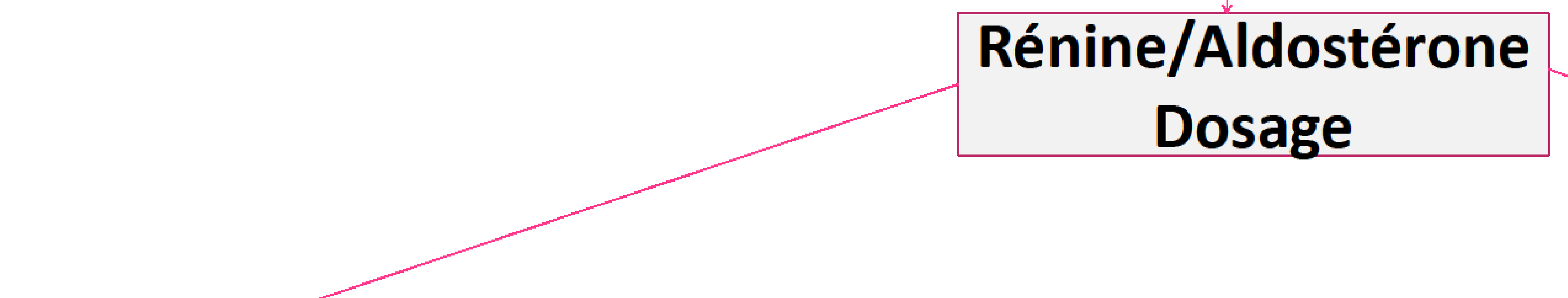

low aldosterone

high renin

low aldosterone
low renin

primary aldosteronism

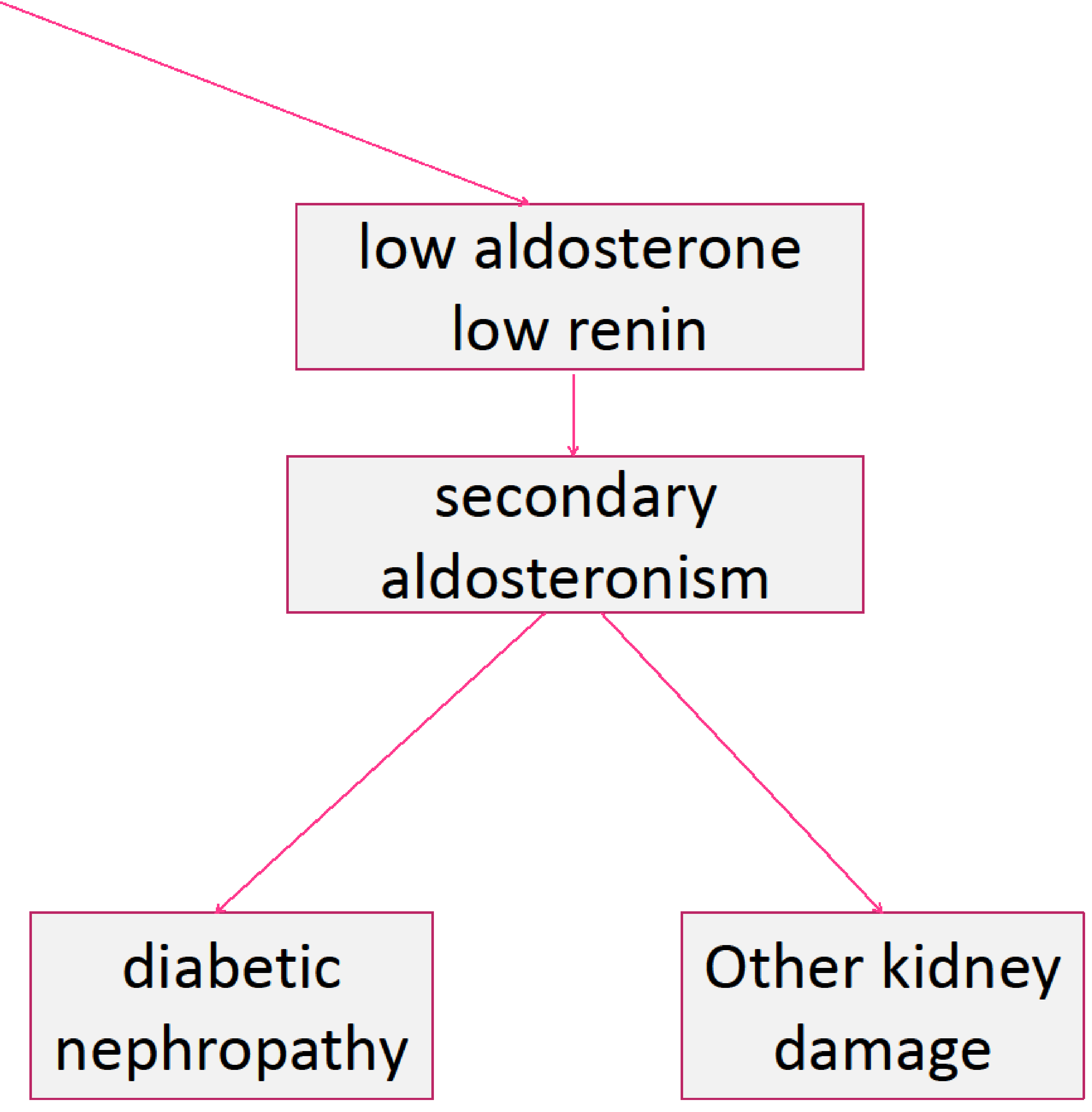

\section{Congenital}

18 hydroxylase 18 Déshydrogénase

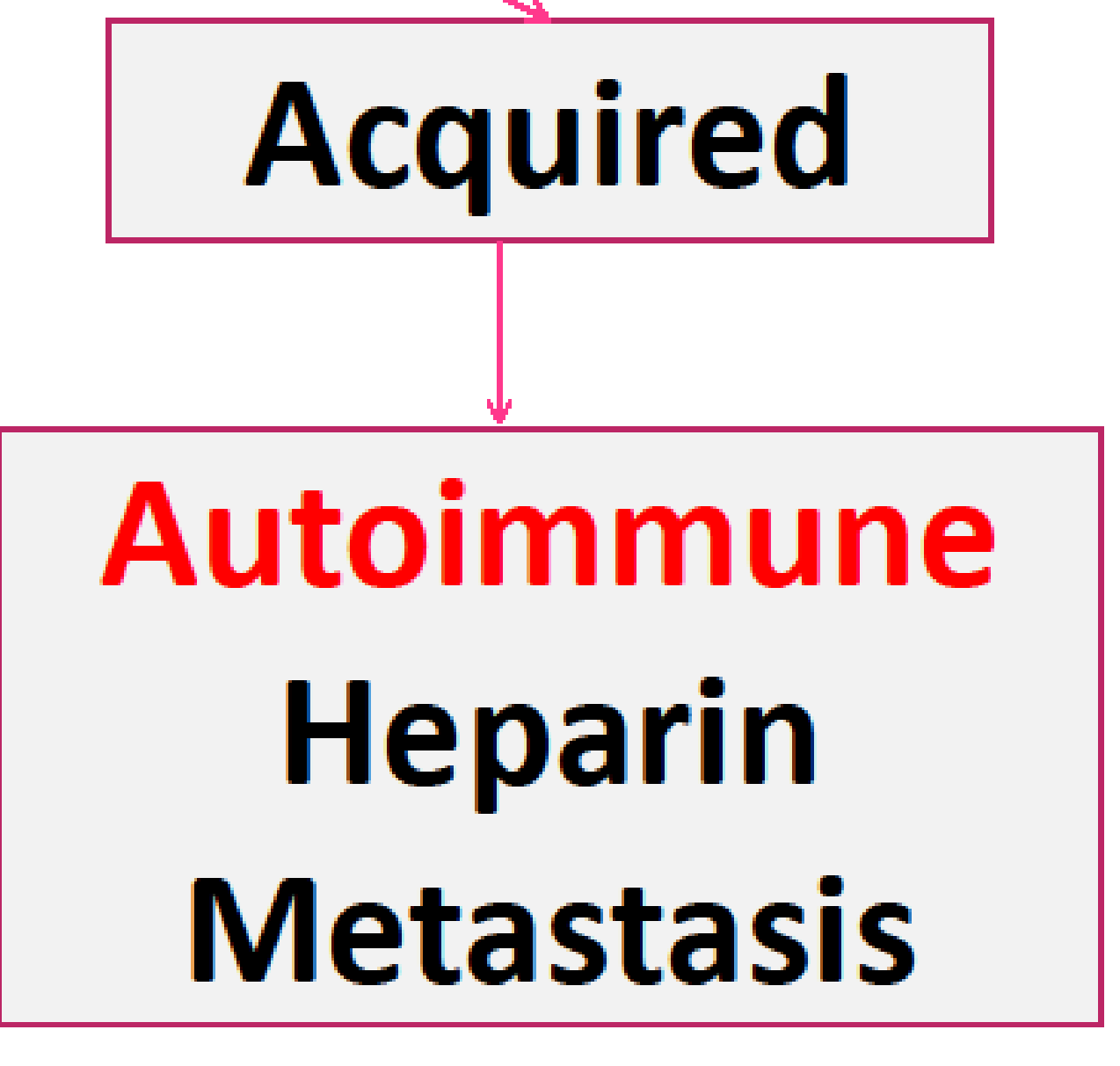

Discussion: Typically hypo aldosteronism is suspected if there is a persistent hyperkalemia, but sometimes it can be revealed by psychiatric troubles due to severe hyponatremia causing infra-radiological cerebral edema as in the illustrated case in who all symptoms resolved after Fludrocortisone. On the etiological side, we have ruled out the obvious causes such as heparin intake, severe diseases, and adrenal metastases. Were also discussed late onset genetic enzyme deficits, but autoimmune etiology was the most probable because of the patient background and positivity to anti $210 \mathrm{Hase}$ anti bodies: $2.2 \mathrm{U} / \mathrm{ml}(\mathrm{n}<1)$.

Conclusion: The isolated primary aldosteronism remains an underestimated cause of hyponatremia; it should be included in the etiological diagnosis of resistant cases to conventional therapy. 